\title{
IDENTIFYING SUITABLE SEGMENTATION PARAMETERS FOR AN OBJECT-BASED IMAGE CLASSIFICATION
}

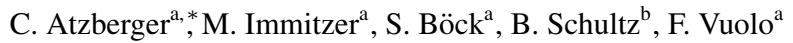 \\ ${ }^{\text {a }}$ Institute of Surveying, Remote Sensing and Land Information (IVFL), University of Natural Resources and Life Sciences, \\ Vienna (BOKU), Peter-Jordan-Straße 82, A-1190 Wien - \\ (clement.atzberger, markus.immitzer, sebastian.boeck, francesco.vuolo)@boku.ac.at \\ ${ }^{\mathrm{b}}$ Instituto Nacional de Pesquisas Espaciais (INPE), Divisão de Sensoriamento Remoto (DSR) - schultz@dsr.inpe.br
}

KEY WORDS: Unsupervised Segmentation Evaluation, Parameter Optimization, Global Score

\begin{abstract}
:
Only well-chosen segmentation parameters ensure optimum results of object-based image classifications. Manually defining suitable parameter sets can be a time-consuming approach, not necessarily leading to optimum results. Moreover, the manual approach is obviously subjective. An automated evaluation approach is needed to reduce human intervention and to provide objective criteria for ranking different parameter sets.

In this work, we test three different ways to find the optimum segmentation.

(i) We used a supervised approach integrating the segmentation and classification tasks. The segmentation is optimized directly with respect to the overall accuracy of the subsequent classification.

(ii) Using the global score value considering within- and between segment heterogeneity, we run an unsupervised segmentation optimization to find the best segmentation parameters.

(iii) Using manually delineated objects we calculate discrepancy measurements like euclidean distance between automatic generated objects and the manual delineated objects in a supervised segmentation evaluation.

For all approaches, we present fully autonomous workflows for supervised and unsupervised object-based classification, combining image segmentation, segmentation evaluation and image classification. Starting from a fixed set of randomly selected and manually delineated training samples, suitable segmentation parameters are automatically identified. Finally, we compare the results of the three different approaches.
\end{abstract}

\footnotetext{
${ }^{*}$ Corresponding author
} 